\title{
Impact of season and geography on CompEx Asthma: a composite end-point for exacerbations
}

\author{
Alexandra Jauhiainen ${ }^{1,8}$, Lieke E.J.M. Scheepers ${ }^{1,8}$, Anne L. Fuhlbrigge ${ }^{2}$, \\ Tim Harrison ${ }^{3}$, James Zangrilli ${ }^{4}$, Esther Garcia Gil ${ }^{5}$, Per Gustafson ${ }^{6}$, \\ Malin Fagerås ${ }^{6}$ and Carla A. Da Silva ${ }^{7}$
}

Affiliations: ${ }^{1}$ BioPharma Early Biometrics and Statistical Innovation, Data Science \& Al, BioPharmaceuticals R\&D, AstraZeneca, Gothenburg, Sweden. ${ }^{2}$ Pulmonary Sciences and Critical Care, Dept of Medicine, University of Colorado School of Medicine, Denver, CO, USA. ${ }^{3}$ Nottingham NIHR Biomedical Research Centre, Respiratory Medicine, University of Nottingham, Nottingham, UK. ${ }^{4}$ BioPharmaceuticals Medical, AstraZeneca, Gaithersburg, MD, USA. ${ }^{5}$ BioPharmaceuticals Medical, AstraZeneca, Barcelona, Spain. ${ }^{6}$ BioPharmaceuticals Medical, AstraZeneca, Gothenburg, Sweden. ${ }^{7}$ Early Respiratory and Immunology Clinical Development, BioPharmaceuticals R\&D, AstraZeneca, Gothenburg, Sweden. ${ }^{8}$ Shared first authors.

Correspondence: Carla Da Silva, Early Respiratory and Immunology Clinical Development, BioPharmaceuticals R\&D, AstraZeneca, Pepparedsleden 1, 43153 Mölndal, Sweden. E-mail: carla.dasilvala astrazeneca.com

\section{ABSTRACT}

Background: CompEx Asthma, a novel composite end-point combining severe exacerbations (SevEx) with asthma-worsening events, was recently developed. Further characterisation of CompEx Asthma is needed to illustrate the applicability of this end-point. The objective was to evaluate CompEx Asthma as a rate end-point to determine how seasonal and geographical factors impact this novel outcome.

Methods: Seven 24-56-week randomised controlled trials of budesonide/formoterol (BUD/FORM) and benralizumab were analysed. Annualised event rates (AERs) and treatment effects (hazard ratio (HR)) were analysed with Poisson and Andersen-Gill models, respectively. Seasonality was analysed by month and five geographical regions were evaluated.

Results: The studies included 10815 patients (63\% female, mean age 42-49 years). CompEx Asthma AER mirrored seasonal variations in SevEx AER. CompEx Asthma AERs were higher versus SevEx in BUD/FORM and benralizumab trials (range 2.7-4.5-fold and 1.3-2.0-fold increase, respectively) and were less variable versus SevEx between regions (ratios of greatest:smallest AERs: 1.36 for CompEx versus 2.28 for SevEx (BUD/ FORM); 1.81 for CompEx versus 2.22 for SevEx (benralizumab)). Treatment effects for CompEx Asthma and SevEx were generally similar across regions and months. However, in Eastern Europe, where SevEx rates were lowest, treatment effect was greater with CompEx Asthma versus SevEx, reaching statistical significance in the benralizumab studies (HR (95\% CI): 0.67 (0.53-0.85) versus 0.87 (0.65-1.15)).

Conclusion: This study confirmed the reliability of CompEx Asthma as a rate end-point and allowed detection of variations in seasonal SevEx rates, reduction of variation in rates across regions and potential greater sensitivity to treatment effects.

@ERSpublications

CompEx Asthma is a valid end-point that reflects the treatment efficacy observed on SevEx when studying recurrent events and may enable smaller, 3-month clinical trials factoring in seasonal variation https://bit.ly/3jPI894

Cite this article as: Jauhiainen A, Scheepers LEJM, Fuhlbrigge AL, et al. Impact of season and geography on CompEx Asthma: a composite end-point for exacerbations. ERJ Open Res 2020; 6: 00246-2020 [https://doi.org/10.1183/23120541.00246-2020].

This article has supplementary material available from openres.ersjournals.com

Received: 4 May 2020 | Accepted after revision: 21 July 2020

Copyright $\odot$ ERS 2020. This article is open access and distributed under the terms of the Creative Commons Attribution Non-Commercial Licence 4.0 . 


\section{Introduction}

Asthma is a heterogeneous chronic inflammatory disease of the airways punctuated by recurring episodes of worsening control [1]. Severe exacerbations (SevEx), defined as asthma worsening leading to oral corticosteroid (OCS) treatment, emergency room visit and/or hospitalisation, occur across the spectrum of asthma severity and represent a significant burden to patients and the community [1,2]. Due to their infrequent nature, clinical trials using SevEx as an end-point typically require observation periods of $\geqslant 6$ months and large sample sizes to gain sufficient statistical power to enable comparison between interventions [3-6]. Consequently, SevEx are rarely used as the primary outcome measure in the early phases of drug development. In a recent post hoc analysis, a novel composite end-point for exacerbations (CompEx) was defined that captures clinically relevant asthma-worsening episodes based on combination of diary events (worsening in daily peak expiratory flow (PEF), asthma symptoms and reliever medication use) plus SevEx events. Time-to-first event analysis suggested that CompEx could allow for shorter trials with fewer patients while maintaining the ability to detect treatment effects equivalent to that of SevEx only as an outcome measure [7]. Since the initial publication, the CompEx end-point has also been adapted to COPD (COPDCompEx), so we will refer to it herein as CompEx Asthma.

SevEx rates in patients of all ages are influenced by seasonal and geographical factors [8-10]. Seasonal peaks are thought to be attributable to viral respiratory infections, which are responsible for $50-75 \%$ of exacerbations in adult patients $[11,12]$. Differences in asthma exacerbation rates are also observed between geographic regions, with consistently fewer exacerbations recorded in Eastern Europe compared with North America, Western Europe and Asia [13, 14]. This could be due to a range of factors including differences in climate, aeroallergen and air pollution levels, socioeconomic differences and variations in diagnostic and asthma management criteria $[15,16]$. Observed regional variations in exacerbation rates may also reflect variation in perceived need for oral prednisolone by patients and healthcare professionals, quality of primary care and accessibility to and cost of treatment, rather than purely clinical differences in the event frequency [17].

To extend the applicability of CompEx Asthma and further characterise this end-point, we undertook a post hoc analysis of seven randomised controlled trials, five of which were used in the initial development of the end-point. We analysed CompEx Asthma as a rate end-point to specifically test the hypotheses that CompEx Asthma behaves similarly to SevEx across geographical region and season of the year, while maintaining the treatment effect demonstrated by SevEx.

\section{Methods}

To evaluate the hypotheses on geographical and seasonal trends for CompEx Asthma and SevEx, we compared the performance of the two end-points in terms of annualised event rate (AER). Treatment effects were examined using Anderson-Gill modelling assumption. We also evaluated the AER and treatment effects on diary events independently, to highlight their contribution to the composite CompEx Asthma end-point. Details of the data used and methods applied follow below.

\section{Study design and data collection}

In this post hoc analysis, data from seven 24- to 56-week randomised clinical trials were analysed, including 10815 patients aged $\geqslant 16$ years with asthma ranging from mild to severe (figure 1 ) $[3-6,13,14$, 18]. The studies were chosen based on two criteria: the five studies of budesonide/formoterol (BUD/ FORM trials) used to initially develop the CompEx Asthma end-point were included to illustrate that CompEx Asthma can also be used as a rate end-point with similar treatment effects (this was illustrated by FuHLBRIgGe et al. 2017 [7] for time-to-first analyses). Second, the size and duration of the studies were important to enable testing of the new hypotheses related to geographical and seasonal trends. The two benralizumab trials were included as new test studies based on similar criteria for treatment duration and study size (supplementary table E1). Further details on the studies used are available in the supplementary appendix.

\section{Definition of CompEx Asthma, diary events and SevEx}

The CompEx Asthma end-point was developed by combining asthma-worsening diary events (defined using diary card variables) with SevEx events. The methodology for defining diary events has been previously described in detail [7]. Briefly, diary events are based on objective measures of worsening in $\mathrm{PEF}$, reliever medication use and worsening of asthma symptoms assessed morning and evening in daily diaries (six variables in total). Asthma-worsening diary events were defined as either two diary variables reaching a predefined threshold change from baseline for at least two consecutive days, or deterioration in all six variables over at least a 5-day period combined with a threshold change in at least one variable (further detail provided in the supplementary appendix). 


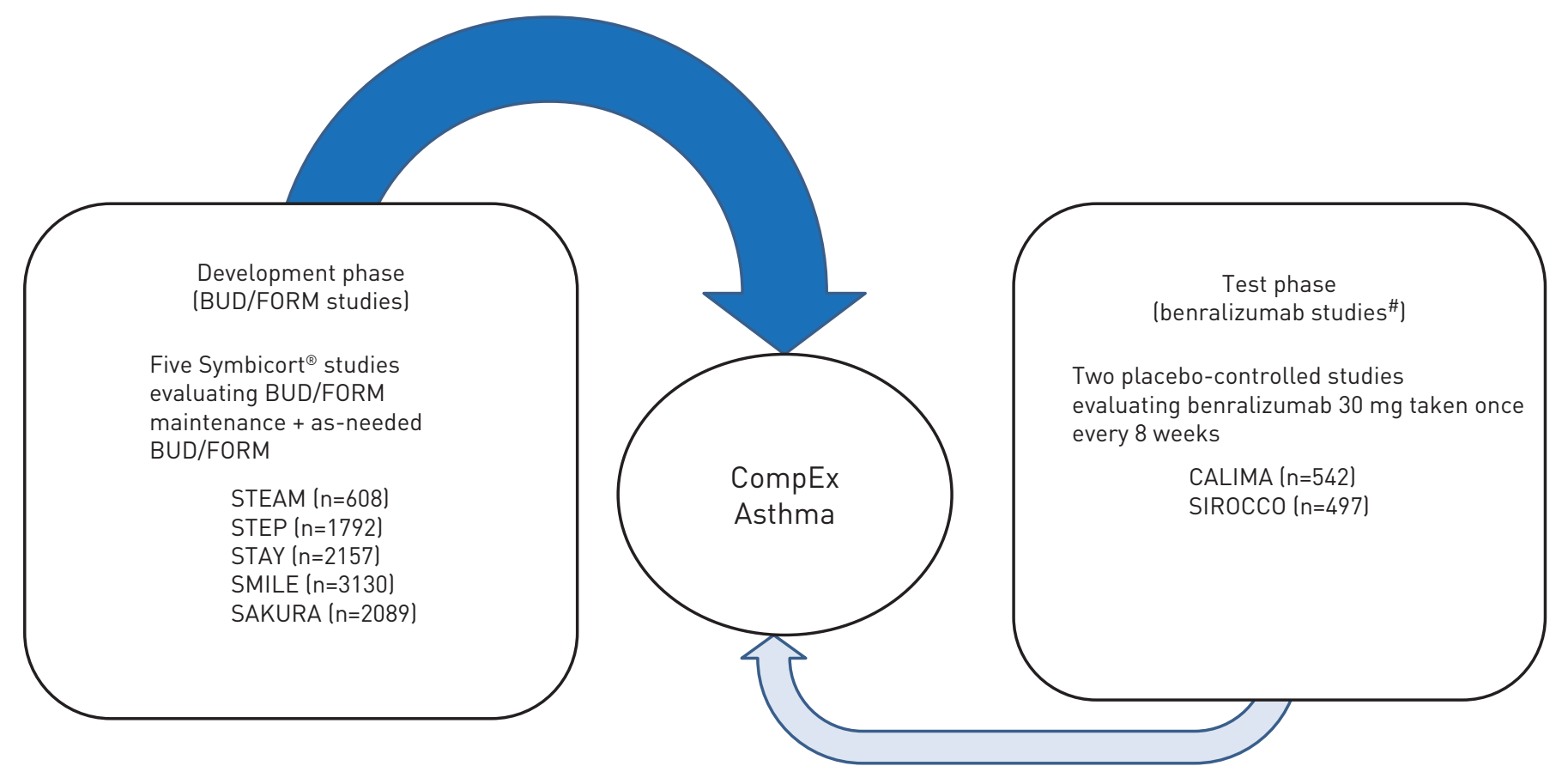

FIGURE 1 Breakdown of the studies used in the development and test phases of the CompEx Asthma analyses. All studies in patients aged

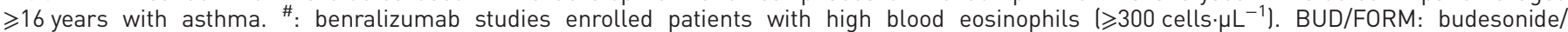
formoterol; CompEx: composite end-point for exacerbations.

The definition of SevEx events was consistent across studies and based on a requirement for OCS treatment and/or hospitalisation or emergency treatment for asthma. The per-protocol definition of recurrent SevEx events (i.e. events considered to be separate worsening events) differed slightly between the BUD/FORM studies, with gaps between events of 0 to 2 days. In the benralizumab studies, a SevEx event was considered recurrent when the gap between events exceeded the per-protocol definition (i.e. 7 days).

To enable rate type analyses for CompEx Asthma events, recurrent (i.e. separate) diary events needed to be defined and identified. This was done by scoring days across a patient's diary dataset according to the diary event worsening criteria, with an event continuing until the criteria were no longer fulfilled for a pre-defined number of days (gap) in a row. CompEx Asthma events were then defined as the occurrence of either a diary event or SevEx, with the start/end of an event marked as the first/last day that the criteria for either a diary event or SevEx were fulfilled. In order to determine how to define separate events, we conducted an analysis to assess gaps of 1-, 7- and 10-day duration, where subsequent events that fall within the specified gap are collapsed into a single event. Our analysis found that gaps of 7 and 10 days performed better than a gap of 1 day, and produced similar observed treatment effect for both CompEx Asthma and SevEx (supplementary figure E1). Therefore, we chose to use a gap of 7 days for our definition of CompEx Asthma, in line with the American Thoracic Society/European Respiratory Society statement [2] and the definition of separate SevEx events used in the benralizumab studies [13, 14].

\section{Statistical analyses}

All BUD/FORM studies were pooled for the AER analyses, while data from selected treatment arms from STAY, SMILE and SAKURA were pooled to investigate treatment effects as these studies had similar trial design which enabled assessment of a common treatment comparison. The benralizumab studies were pooled to assess treatment effects and AERs. AERs were estimated using a Poisson model for all analyses, using $\log$ (time-at-risk) as an offset variable. A full time-at-risk analysis was used in the Poisson model, including time in the event and gap time between events. Treatment effects were initially evaluated using three different models for analysing recurrent events: a negative binomial (NB) model, an Andersen-Gill (AG) time-to-recurrent event model and a frailty time-to-recurrent event model (supplementary figure E1). Further details of the models are described in the supplementary appendix.

The treatment effect for CompEx Asthma was compared to the treatment effect observed on SevEx and diary events alone with all models. Both the AG and NB models outperformed the frailty model, which 
was not explored further. Treatment effects for both the NB and AG models are compared in supplementary figure E2. The NB and AG models are limited by the assumption of constant risk of events during follow-up, but the AG model benefits from taking both the timing and ordering of events into account and allowing for the inclusion of time-varying covariates (useful for seasonality analyses). Accordingly, we present results from this model (fitted with a robust variance estimate to avoid Type I error inflation) in the main manuscript and results from the NB model in the supplementary appendix.

The performance of CompEx Asthma in response to seasonal variation was evaluated on a month-by-month basis [9]. To compensate for the differing seasons in the two hemispheres, a number was assigned to each month and mapped according to the northern and southern hemispheres (i.e. January is month 1 in the northern hemisphere, while July is month 1 in the southern hemisphere).

The performance of CompEx Asthma according to geography was analysed by categorising regions as follows: Asia, Eastern Europe, Western Europe, North America and rest of the world (RoW). To evaluate the performance of CompEx Asthma according to geographical variation, AG models were fitted per region. In the seasonal analysis, month was included as a time-varying covariate and treatment effects were extracted using a treatment-month interaction. Treatment effect comparisons were evaluated using Wald-type p-values and confidence intervals (CIs) across all analyses, only using data from the separate treatment arms in each comparison. Additional analyses were performed using the NB model, presented in supplementary figures E3 and E4.

\section{Results}

\section{Patient demographics}

The study population for the BUD/FORM trials included 9776 patients who were predominantly female (63\%), with a mean age ranging from 42 to 46 years, a mean forced expiratory volume in $1 \mathrm{~s}\left(\mathrm{FEV}_{1}\right)$ $70-75 \%$ predicted and prescribed low- to high-dose inhaled corticosteroids (ICS). A total of 1039 patients with high blood eosinophils $\left(\geqslant 300\right.$ cells $\left.\mu \mathrm{L}^{-1}\right)$ from the $\mathrm{q} 8 \mathrm{w}$ treatment arm were analysed in the benralizumab trials. Patients were predominantly female (63\%), with a mean age of 49 years, a mean $\mathrm{FEV}_{1}$ $55-58 \%$ predicted and prescribed medium- to high-dose ICS (supplementary table E2).

\section{Overall annualised event rates}

In the pooled BUD/FORM trials, overall mean CompEx Asthma AER was 3.1-times higher versus SevEx (0.96 versus 0.31; considering all treatment arms) (figure 2a). In the benralizumab trials, mean CompEx Asthma AER was 1.57-times higher versus SevEx (1.45 versus 0.92 ; considering all treatment arms) (figure $2 \mathrm{~b}$ ).

\section{CompEx Asthma according to seasons}

Most patients were recruited and randomised in summer (months 6, 7 and 8; 35.9\%) and autumn (months 9, 10 and $11 ; 31.5 \%$ ) in the BUD/FORM studies, but all trials included patients recruited in all months; spring (months 3, 4 and 5; 15.5\%) and winter (months 12, 1 and 2; 17.1\%) (supplementary figure E5). In the benralizumab studies, recruitment was more evenly distributed across the months (spring: 22.0\%; summer: 24.4\%; autumn: $27.1 \%$; winter: $26.4 \%$ ) (supplementary figure E5). In the pooled BUD/ FORM trials, trends in AERs for CompEx Asthma, SevEx and diary events followed a similar seasonal pattern on a month-by-month basis for all treatment arms (figure 3a; supplementary table E3). Overall, AERs were higher in the winter months (AER range: 0.99-1.23 and 0.36-0.44 for CompEx Asthma and SevEx, respectively) and lower in the summer months (AER range: $0.79-0.88$ and $0.24-0.26$, respectively) (figure 3a; supplementary table E3). The trends in AERs observed in the BUD/FORM studies for CompEx Asthma and SevEx were confirmed in the pooled benralizumab trials; higher in the winter, lower in the summer (AER range in winter: 1.53-2.03 and 0.99-1.23; AER range in summer: 1.04-1.25 and 0.58-0.76 for CompEx Asthma and SevEx, respectively) (figure 3b; supplementary table E4). Across all seasons, AERs for SevEx were considerably lower than diary events in the BUD/FORM trials, while AERs were similar between SevEx and diary events in the benralizumab trials (figure $3 \mathrm{a}$ and b, respectively; supplementary table E4).

The HRs for observed treatment effect on CompEx Asthma were consistent across months in all studies analysed (supplementary figure E6). In addition, season-associated variability in treatment effect was similar with CompEx Asthma and SevEx in the pooled data from STAY, SMILE and SAKURA and in the benralizumab trials (supplementary figure E6).

\section{CompEx Asthma according to geography}

The majority of patients in this analysis were recruited from the northern hemisphere (83\%). The regional distribution of subjects in each study has been tabulated in the supplementary material (supplementary table E5). In the pooled BUD/FORM studies, AERs for CompEx Asthma were less variable across regions 

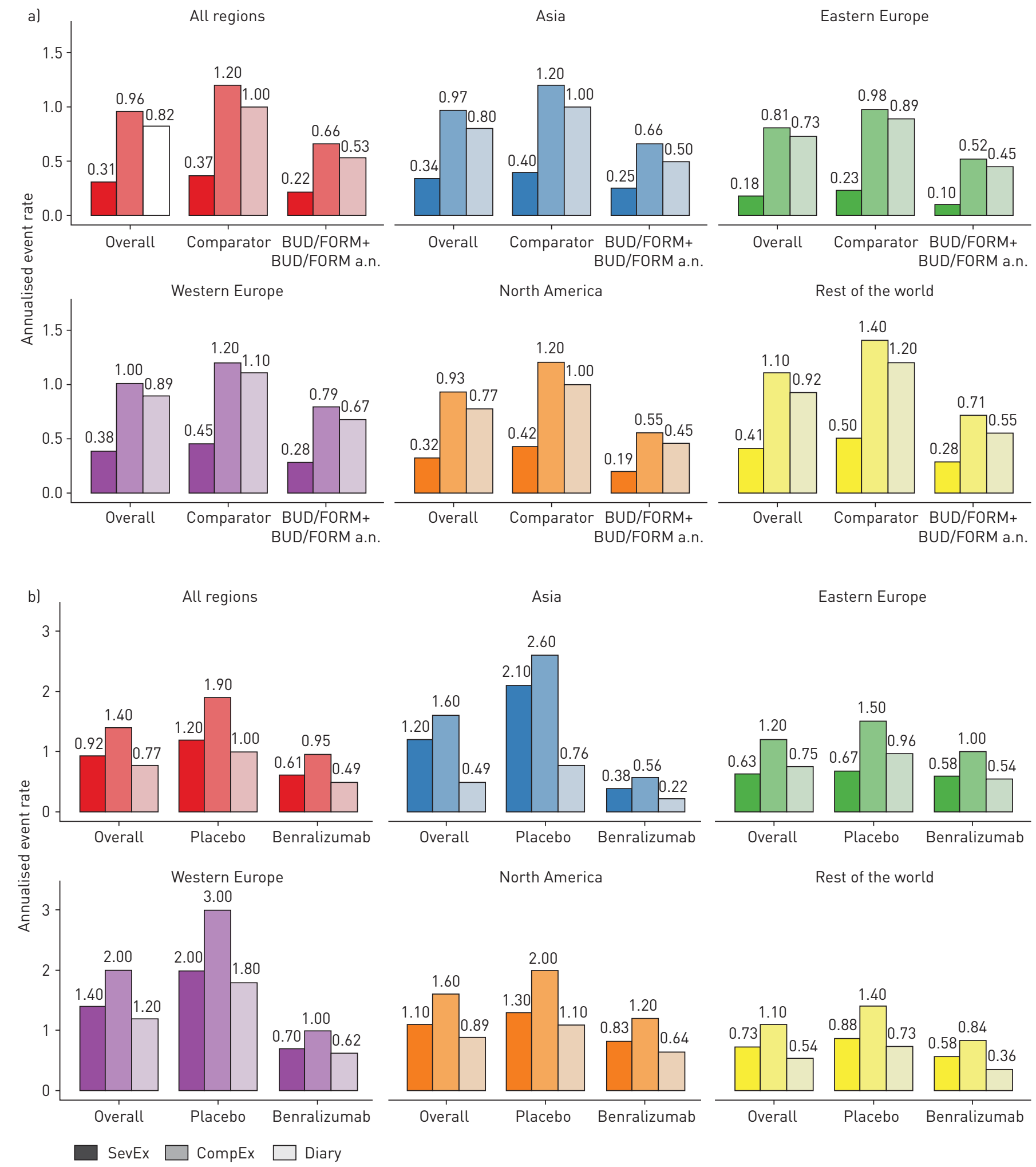

North America

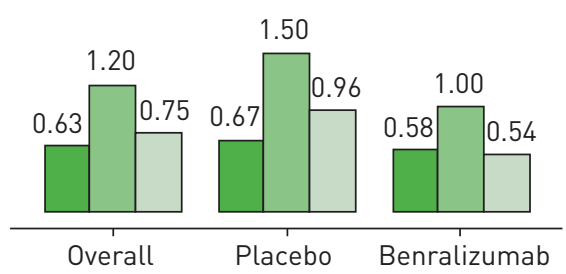

FIGURE 2 Regional pattern of annualised event rates for SevEx, CompEx Asthma and diary events across treatment arms in (a) the pooled BUD/ FORM studies; and (b) the pooled benralizumab studies (see supplementary tables E6-E7 for full dataset and variability). Randomisation ratio: 1:1 for all treatment arms. Number of patients per region: BUD/FORM studies: Asia, $n=2666$; Eastern Europe, $n=2697$; Western Europe, $n=2537$; North America, $n=455$; RoW, $n=1141$. Benralizumab studies: Asia, $n=135$; Eastern Europe, $n=339$; Western Europe, $n=173$; North America, $n=171$; RoW, n=221. a.n.: as-needed; BUD/FORM: budesonide/formoterol; CompEx: composite end-point for exacerbations; SevEx: severe exacerbations; RoW: rest of world. 
a)

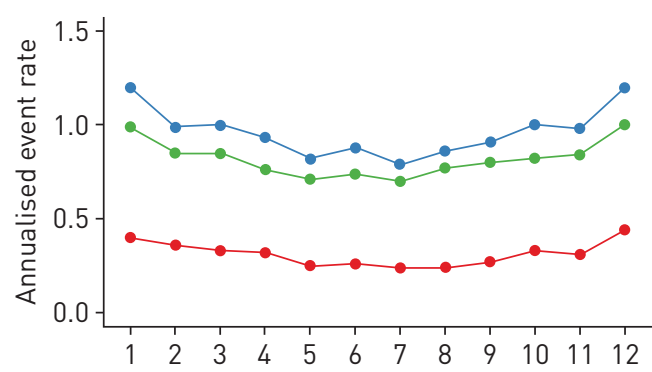

b)

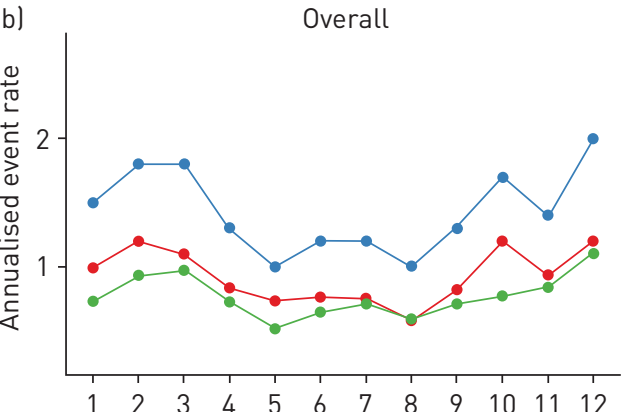

$\bullet$ SevEx $\rightarrow$ CompEx $\rightarrow$ Diary
Comparator
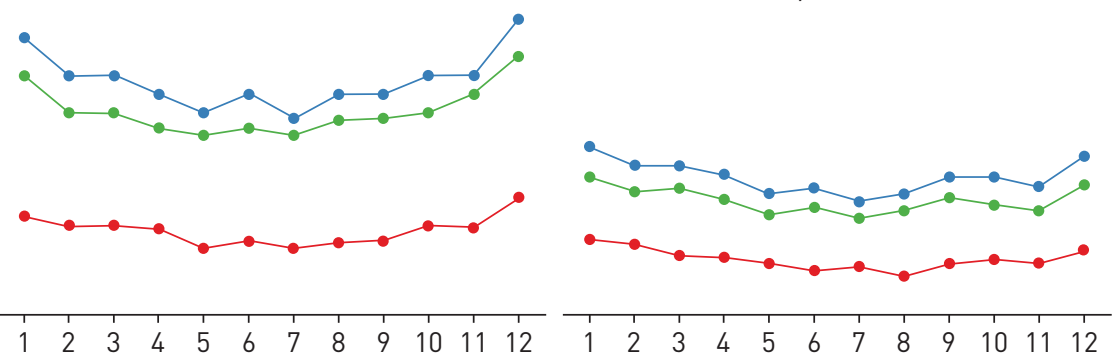

BUD/FORM

+BUD/FORM a.n.

FIGURE 3 Seasonality patterns (by month) of annualised event rates for SevEx, CompEx Asthma and diary events across treatment arms in (a) the pooled BUD/FORM studies; and (b) the pooled benralizumab studies (see supplementary tables E3-E4 for full dataset and variability). Spring months: 3, 4 and 5; summer months: 6, 7 and 8; autumn months: 9, 10 and 11; winter months; 12, 1 and 2. a.n.: as-needed; BUD/FORM: budesonide/formoterol; CompEx: composite end-point for exacerbations; SevEx: severe exacerbations.

versus SevEx; the ratio of the highest to the lowest AER for CompEx Asthma was 1.36 (highest/lowest AER: 1.1/0.81) and 2.28 for SevEx (highest/lowest AER: 0.41/0.18) (figure 2a; supplementary table E6). AERs for CompEx Asthma and SevEx were highest in RoW (CompEx Asthma: 1.10; SevEx: 0.41) and lowest in Eastern Europe (CompEx Asthma: 0.81; SevEx: 0.18) (figure 2a; supplementary table E6). AERs with CompEx Asthma ranged from 2.7 to 4.5 times higher versus SevEx, with notably higher AERs in regions where SevEx rates were low; 4.5 times higher in Eastern Europe (low SevEx) versus 2.7 times higher in RoW (high SevEx) (figure 2a; supplementary table E6). Similar to the BUD/FORM studies, AERs for CompEx Asthma were less variable across regions versus SevEx in the benralizumab studies; ratios of greatest/smallest AERs for CompEx Asthma and SevEx were 1.81 and 2.22, respectively (AER ranges; CompEx Asthma: 1.13-2.00; SevEx: 0.63-1.37) (figure 2b; supplementary table E7). AERs with CompEx Asthma ranged from 1.3 to 2.0 times higher versus SevEx in the benralizumab studies. As with the BUD/FORM studies, CompEx Asthma AERs were 2.0 times higher versus SevEx in Eastern Europe where SevEx AERs were low (CompEx Asthma: 1.24; SevEx: 0.63). Diary event AERs were considerably higher than SevEx AERs in the BUD/FORM trials for all regions studied, whereas AERs for diary events were similar or lower than SevEx in the benralizumab studies (figure 2; supplementary tables E6 and E7).

The HR for observed treatment effect was highly variable across regions for CompEx Asthma and SevEx, ranging from $0.37-0.62$ and $0.41-0.66$ (pooled STAY, SMILE and SAKURA) and 0.20-0.67 and 0.17-0.87 (benralizumab studies), respectively (figure 4). The treatment effect observed with SevEx was reflected with CompEx Asthma across all regions in the pooled data from STAY, SMILE and SAKURA and across Asia, Western Europe, North America and RoW in the benralizumab studies (figure 4). In the benralizumab studies, treatment effect in Eastern Europe was greater and less variable with CompEx Asthma, which, in combination with the relatively high CompEx Asthma event rate in this region, led to a statistically significant treatment effect (HR: 0.67; 95\% CI: 0.53-0.85) but not with SevEx (HR: 0.87; 95\% CI: $0.65-1.15$ ) (figure 4b).

\section{Discussion}

This is the first study to evaluate the performance of CompEx Asthma as a rate end-point with analysis of trends across different seasons and geographical regions. We found that CompEx Asthma rates (and diary 

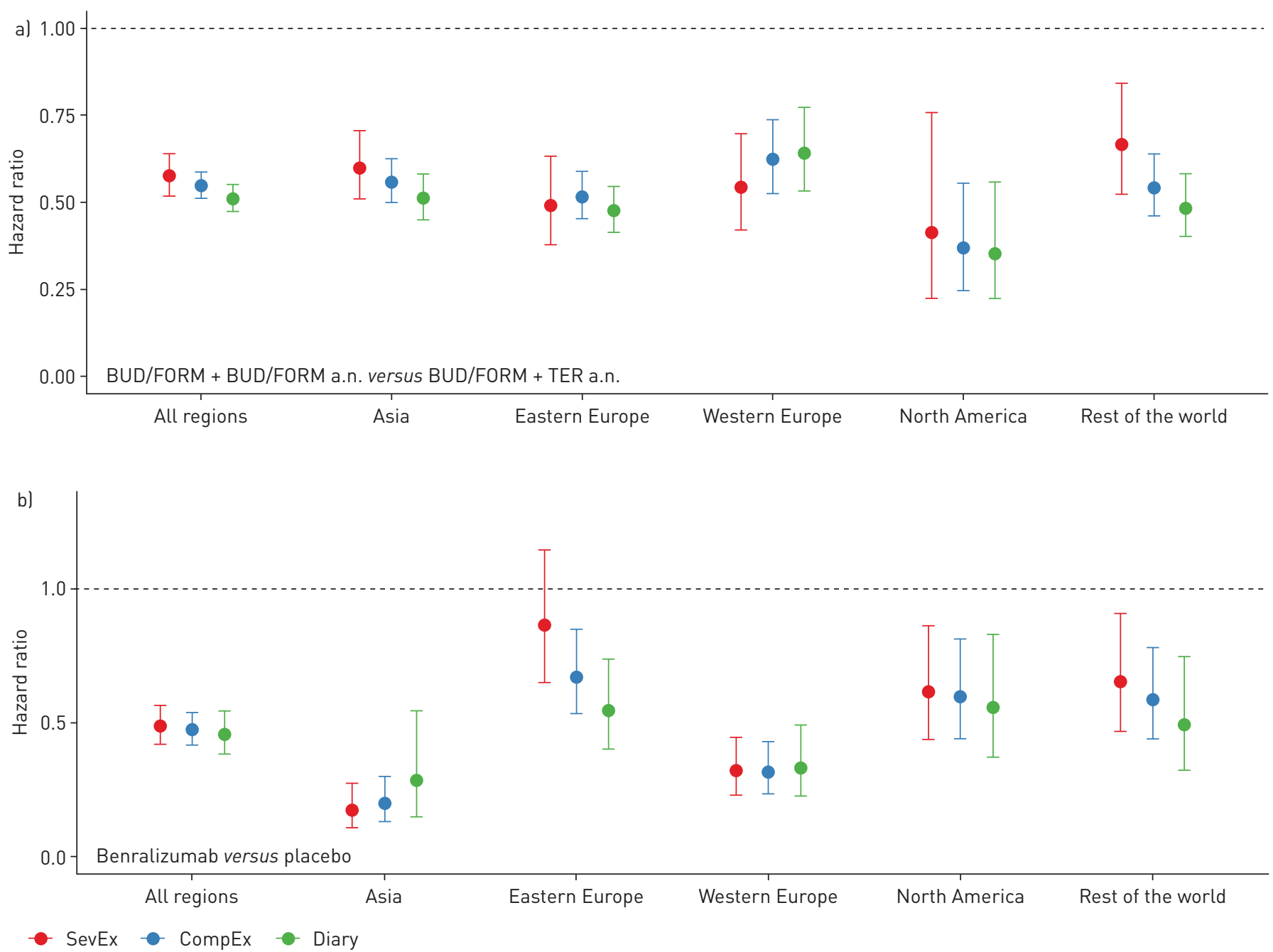

FIGURE 4 Treatment efficacy in regional pattern analysis with SevEx, CompEx Asthma and diary events using the Andersen-Gill model in (a) pooled STAY, SMILE and SAKURA; and (b) the pooled benralizumab studies. Number of patients per region: BUD/FORM studies: Asia, $\mathrm{n}=2666$; Eastern Europe, $n=2697$; Western Europe, $n=2537$; North America, $n=455$; RoW, $n=1141$. Benralizumab studies: Asia, $n=135$; Eastern Europe, $n=339$; Western Europe, $n=173$; North America, $n=171$; RoW, $n=221$. a.n.: as-needed; BUD/FORM: budesonide/formoterol; CompEx: composite end-point for exacerbations; SevEx: severe exacerbations; TER: terbutaline; RoW: rest of world.

events) followed a similar pattern to that observed for SevEx, in line with our hypothesis. Further, although variation in treatment efficacy occurred with both CompEx Asthma and SevEx on a month-by-month basis, we observed less variation in the observed treatment effects (HRs) with CompEx Asthma. It is unclear whether this apparent stability is an intrinsic property of the CompEx Asthma measure or if it reflects the effect of the treatment intervention.

Seasonal patterns of SevEx rates have been reported previously in adults, with winter peaks in both the northern and southern hemispheres, often associated with viral respiratory infections [9]. An exception in this analysis was the STEAM trial, where monthly variability was reported across all study end-points with no apparent seasonal pattern. Small sample size and short study duration (6 months) in this trial may have contributed to this finding.

The hypothesis related to geographical variability with the different end-points was similarly evaluated by first looking at event rates across regions. Event rates were less variable (assessed by the ratio of highest/ lowest AERs) across regions with CompEx Asthma compared with SevEx. The largest difference in AER for CompEx Asthma compared to SevEx was observed in Eastern Europe, a region with consistently low SevEx rates in the clinical trials. For both the BUD/FORM and benralizumab trials in this region, the diary event component of CompEx Asthma seems to compensate for the relatively low SevEx rate. The reason for the low observed SevEx rate in Eastern Europe is not known, but may reflect differences in patient and health care professional use of systemic corticosteroids and threshold for emergency/hospital referrals, rather than true differences in the frequency of exacerbation events. 
The geographical variability was further evaluated by comparing treatment effects (HRs) across regions. The observed treatment effects with CompEx Asthma were similar to the effects observed on SevEx across regions in the BUD/FORM studies, with significant effects both for CompEx Asthma and SevEx. However, in regions with low SevEx event rates in the pooled benralizumab studies, the treatment effects with CompEx Asthma were greater versus SevEx, and CompEx Asthma also reached statistical significance in the region where SevEx failed to do so (Eastern Europe).

Although our data are limited in regions with very low event rates (compared with rates in other regions within the same dataset), the event rate and treatment effect trends in Eastern Europe together indicate that CompEx Asthma appears to adjust for societal and healthcare differences across regions that can confound SevEx results.

AERs with CompEx Asthma were higher compared with SevEx AERs across all studies. However, AERs were considerably higher in the benralizumab versus the BUD/FORM studies, and the difference between CompEx Asthma AERs and SevEx AERs was greater in the BUD/FORM versus the benralizumab studies. The reasons for the disparity between studies is probably related to the fact that by design, the benralizumab studies included patients with a greater frequency of previous exacerbations with an eosinophilic phenotype and low lung function, both risk factors for future exacerbations. The benralizumab studies also employed formal diary-based asthma-worsening alerts that could have driven the higher physician-evaluated SevEx rates and lower diary event rates. In contrast, the BUD/FORM studies did not feature diary-based asthma-worsening alerts, and thus higher diary event rates were reported. These diary events in the BUD/FORM studies could potentially have been unreported SevEx, highlighting the disparity between objective measures (e.g. changes in PEF, reliever use or symptoms) versus physician's discretion in diagnosing a SevEx. Further work is required to investigate the differences in SevEx rates between these study populations.

A major strength of our analysis was the large number of patients included from multiple randomised controlled trials, with diverse study designs, disease severities and multiple active treatment arms and comparators. Further strengths included: the use of a comprehensive dataset for the development of the CompEx Asthma end-point, with a comparable dataset to test the performance of the end-point; and using three models of varying sensitivity for the recurrent event analysis to compare and evaluate the treatment effect across study end-points. While the AG model displayed higher precision versus the NB model, the results and conclusions of CompEx Asthma treatment effect were similar in the NB model analysis.

This study was limited by the post hoc nature of the analyses. CompEx Asthma can only be applied to studies investigating diary events in addition to SevEx, and relies on patients being compliant when filling out diary cards, limiting its use to studies that have accurately captured these data. Furthermore, a limitation of the geographical analyses was that $83 \%$ of the total patient cohort was recruited from the northern hemisphere. More data from southern hemisphere patients would be required in future analyses to confirm our findings in terms of the effects of geographical variation when investigating the CompEx Asthma end-point. In addition, the geographic analyses are potentially limited by the assumption that patients are homogeneous within each individual region.

In the primary CompEx Asthma paper, the novel end-point was specifically designed to enable the development of time- and cost-effective trials with fewer patients in order to evaluate treatment-related reduction in risk of asthma worsening before moving on to phase 3 drug development trials. Here we have demonstrated that CompEx Asthma was generally more sensitive than SevEx when analysing event rates. This reinforces previous findings that CompEx Asthma as an end-point of asthma worsening could permit the acceleration of clinical trials, with a possible $50 \%$ reduction in the size of the patient cohort [7]. Our current analysis demonstrates that with regard to geography, clinical trials using CompEx Asthma as an end-point of asthma worsening could be run anywhere, even in countries with low SevEx rates. CompEx Asthma rates are variable across seasons (as with SevEx), so the optimal recommendation would be to conduct smaller, 3-month clinical trials with seasonal variation factored in. Future direction will focus on gaining regulatory approval for CompEx Asthma as an effective drug development tool and defining the clinical relevance of CompEx Asthma with healthcare professionals.

In conclusion, we have shown that CompEx Asthma event rates are influenced by seasons in a similar way to SevEx, but treatment effects are less variable on a month-by-month basis with CompEx Asthma. CompEx Asthma also demonstrated less variation in event rates across regions and was sensitive to treatment effects even in regions with low SevEx event rates, such as Eastern Europe. This study builds on previous results supporting CompEx Asthma as a valid end-point that reflects the treatment efficacy observed on SevEx when studying recurrent events. 
Support statement: This study was supported by AstraZeneca. The authors would like to thank Samantha Blakemore of inScience Communications, Springer Healthcare Ltd., UK, for providing medical writing support, which was funded by AstraZeneca in accordance with Good Publication Practice (GPP3) guidelines (www.ismpp.org/gpp3). Data underlying the findings described in this manuscript may be obtained in accordance with AstraZeneca's data sharing policy described at https://astrazenecagrouptrials.pharmacm.com/ST/Submission/Disclosure. The funder of the study, AstraZeneca, had a role in study design, data collection, data analysis, data interpretation and writing of the report. The corresponding author had full access to all the data and had final responsibility to submit for publication. Funding information for this article has been deposited with the Crossref Funder Registry.

Author contributions: A. Jauhiainen prepared the data and took the lead on statistical analysis (including generation of figures), data interpretation and review, supplementary material authoring and final approval of the manuscript. L.E.J.M. Scheepers was responsible for data preparation, statistical analysis (including generation of figures), data interpretation and review and final approval of the manuscript. A.L. Fuhlbrigge, T. Harrison, E. Garica Gil, J. Zangrilli and P. Gustafson were responsible for data interpretation and review and final approval of the manuscript. M. Fagerås was responsible for data preparation, data interpretation and review and final approval of the manuscript. C.A. Da Silva provided steering, data interpretation and review and final approval of the manuscript.

Conflict of interest: A. Jauhiainen is a full-time employee of AstraZeneca. L.E.J.M. Scheepers is a full-time employee of AstraZeneca. A.L. Fuhlbrigge is an unpaid consultant to AstraZeneca and reports personal fees and other support from Icon Medical Imaging, and personal fees from Novartis, outside the submitted work. T. Harrison reports nonfinancial support for study analysis and manuscript generation from AstraZeneca during the conduct of the study; and grants, personal fees and nonfinancial support from AstraZeneca, personal fees and nonfinancial support from GSK, and personal fees from Synairgen and Chiesi, outside the submitted work. J. Zangrilli is a full-time employee of AstraZeneca. E. Garcia Gil is a full-time employee of AstraZeneca. P. Gustafson is a full-time employee of AstraZeneca and shareholder. M. Fagerås is a full-time employee of AstraZeneca. C.A. Da Silva is a full-time employee of AstraZeneca.

\section{References}

1 Global Initiative for Asthma (GINA). Global Strategy for Asthma Management and Prevention. 2019. https:// ginasthma.org/reports/ Date last accessed: 11/07/2019.

2 Reddel HK, Taylor DR, Bateman ED, et al. An official American Thoracic Society/European Respiratory Society statement: asthma control and exacerbations: standardizing endpoints for clinical asthma trials and clinical practice. Am J Respir Crit Care Med 2009; 180: 59-99.

3 Atienza T, Aquino T, Fernandez M, et al. Budesonide/formoterol maintenance and reliever therapy via Turbuhaler versus fixed-dose budesonide/formoterol plus terbutaline in patients with asthma: phase III study results. Respirology 2013; 18: 354-363.

4 O'Byrne PM, Bisgaard H, Godard PP, et al. Budesonide/formoterol combination therapy as both maintenance and reliever medication in asthma. Am J Respir Crit Care Med 2005; 171: 129-136.

5 Rabe KF, Atienza T, Magyar P, et al. Effect of budesonide in combination with formoterol for reliever therapy in asthma exacerbations: a randomised controlled, double-blind study. Lancet 2006; 368: 744-753.

6 Rabe KF, Pizzichini E, Stallberg B, et al. Budesonide/formoterol in a single inhaler for maintenance and relief in mild-to-moderate asthma: a randomized, double-blind trial. Chest 2006; 129: 246-256.

7 Fuhlbrigge AL, Bengtsson T, Peterson S, et al. A novel endpoint for exacerbations in asthma to accelerate clinical development: a post hoc analysis of randomised controlled trials. Lancet Respir Med 2017; 5: 577-590.

8 Larsen K, Zhu J, Feldman LY, et al. The annual September peak in asthma exacerbation rates. Still a reality? Ann Am Thorac Soc 2016; 13: 231-239.

9 Reddel HK, Jenkins C, Quirce S, et al. Effect of different asthma treatments on risk of cold-related exacerbations. Eur Respir J 2011; 38: 584-593.

10 Wisniewski JA, McLaughlin AP, Stenger PJ, et al. A comparison of seasonal trends in asthma exacerbations among children from geographic regions with different climates. Allergy Asthma Proc 2016; 37: 475-481.

11 Jackson DJ, Johnston SL. The role of viruses in acute exacerbations of asthma. J Allergy Clin Immunol 2010; 125: 1178-1187. quiz 1188-1179.

12 Jackson DJ, Sykes A, Mallia P, et al. Asthma exacerbations: origin, effect, and prevention. J Allergy Clin Immunol 2011; 128: 1165-1174.

13 Bleecker ER, FitzGerald JM, Chanez P, et al. Efficacy and safety of benralizumab for patients with severe asthma uncontrolled with high-dosage inhaled corticosteroids and long-acting beta2-agonists (SIROCCO): a randomised, multicentre, placebo-controlled phase 3 trial. Lancet 2016; 388: 2115-2127.

14 FitzGerald JM, Bleecker ER, Nair P, et al. Benralizumab, an anti-interleukin-5 receptor alpha monoclonal antibody, as add-on treatment for patients with severe, uncontrolled, eosinophilic asthma (CALIMA): a randomised, double-blind, placebo-controlled phase 3 trial. Lancet 2016; 388: 2128-2141.

15 Fuhlbrigge A, Peden D, Apter AJ, et al. Asthma outcomes: exacerbations. J Allergy Clin Immunol 2012; 129: S34-S48.

16 Johnston NW, Sears MR. Asthma exacerbations. 1: epidemiology. Thorax 2006; 61: 722-728.

17 Kumana CR, So SY, Li KY, et al. Pattern of anti-asthmatic drug utilization in Hong Kong compared to other parts of the world. Respir Med 1989; 83: 343-348.

18 Scicchitano R, Aalbers R, Ukena D, et al. Efficacy and safety of budesonide/formoterol single inhaler therapy versus a higher dose of budesonide in moderate to severe asthma. Curr Med Res Opin 2004; 20: 1403-1418. 